\title{
Synthesis of Benzothiophene Carboxamide Derivatives and their Pharmacological Evaluation as Potent Antihypertriglyceridemic Agents in Rats
}

\author{
Ghassan Abu Sheikha ${ }^{\mathrm{a}}$, Buthina Hussin ${ }^{\mathrm{b}}$, Yusuf Al-Hiari ${ }^{\mathrm{b}}$, Tariq Al-Qirim ${ }^{\mathrm{a}, *}$, \\ and Ghassan Shattat ${ }^{\mathrm{a}}$ \\ a Faculty of Pharmacy, Al-Zaytoonah Private University, Amman 11733, Jordan. \\ Fax: 009626 4291432. E-mail: qirim70@yahoo.com \\ b Faculty of Pharmacy, University of Jordan, Amman, Jordan \\ * Author for correspondence and reprint requests \\ Z. Naturforsch. 66c, 93-103 (2011); received July 1/November 8, 2010
}

Benzothiophene carboxamide derivatives of aminobenzophenone, aminopyridine, aminobenzimidazole, and aniline derivatives (compounds 1-9) were synthesized and compounds $3,6,7,8$, and 9 tested in vivo for their hypolipidemic activity. Compounds $\mathbf{1}-\mathbf{8}$ were prepared adopting the fusion process at $130-150{ }^{\circ} \mathrm{C}$ between benzothiophene-2-carbonyl chloride and aminobenzophenones, aminopyridine, and anilines, respectively, and were obtained in high yield, while compound 9 was obtained from the reaction of benzothiophene acyl chloride with aminobenzimidazole in DMF at $160^{\circ} \mathrm{C}$. At a dose of $15 \mathrm{mg} / \mathrm{kg}$ body weight compounds 6, 7, and 9 significantly reduced plasma triglyceride levels in Triton WR-1339-induced hyperlipidemic rats in comparison to control rats. Furthermore, they significantly increased high-density lipoprotein levels. It is therefore reasonable to assume that compounds 6, 7, and $\mathbf{9}$ may have a promising potential in the treatment of hyperlipidemia and atherosclerosis.

Key words: Antihyperglyceridemia, Benzothiophene Carboxamide, Aminobenzimidazole

\section{Introduction}

There is a great deal of experimental, epidemiological, and clinical evidence suggesting that hyperlipidemia plays an important role in the pathogenesis of atherosclerosis and cardiovascular diseases (Goldstein et al., 1973; Frishman, 1998). This lipid metabolism disorder is characterized by elevated concentrations of circulating lipids including triglycerides, total cholesterol, very low-density lipoprotein, and low-density lipoprotein (Raasch, 1988). Hyperlipidemia is usually asymptomatic and is regularly detected during routine screening. The increased incidence of cardiovascular disease (CVD) among patients with elevated concentrations of circulating lipids has led to increased recognition of hyperlipidemia as important targets of therapy.

In the course of the present study, Triton WR1339-induced hyperlipidemic rats were used as an experimental model to investigate the potential activity of some synthetic hypolipidemic agents. Previous studies showed that a single parenteral administration of Triton WR-1339 to adult rats produces hyperlipidemia in which cholesterol, triglycerides, and phospholipids levels increase to a maximum within about 20 hours and decrease thereafter (Schurr et al., 1972). This activity of Triton WR-1339 was found to be due to its inhibitory effect on lipoprotein lipase (Schotz et al., 1957).

Currently hyperlipidemia is treated with different groups of drugs including fibrates and their derivatives (such as bezafibrate) (Frick et al., 1987). Fibric acids enhance the fatty acid catabolism and accordingly reduce plasma lipid levels, predominantly triglyceride levels (Rubins et al., 1999). The main mechanism was found to be through decreasing the synthesis of apoC-III and increasing the activity of lipoprotein lipase, which together enhance the clearance of circulating triglyceride-rich lipoproteins (Schoonjans et al., 1996).

Benzothiophene and its derivatives have been reported to show activity in treating various medical conditions, such as hyperlipidemia, breast cancer, and uterus cancer (Charles et al., 1984). On the other hand, other groups of compounds such as benzophenone, aminopyridine, and ami- 


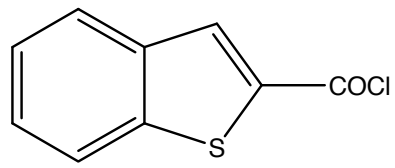

10

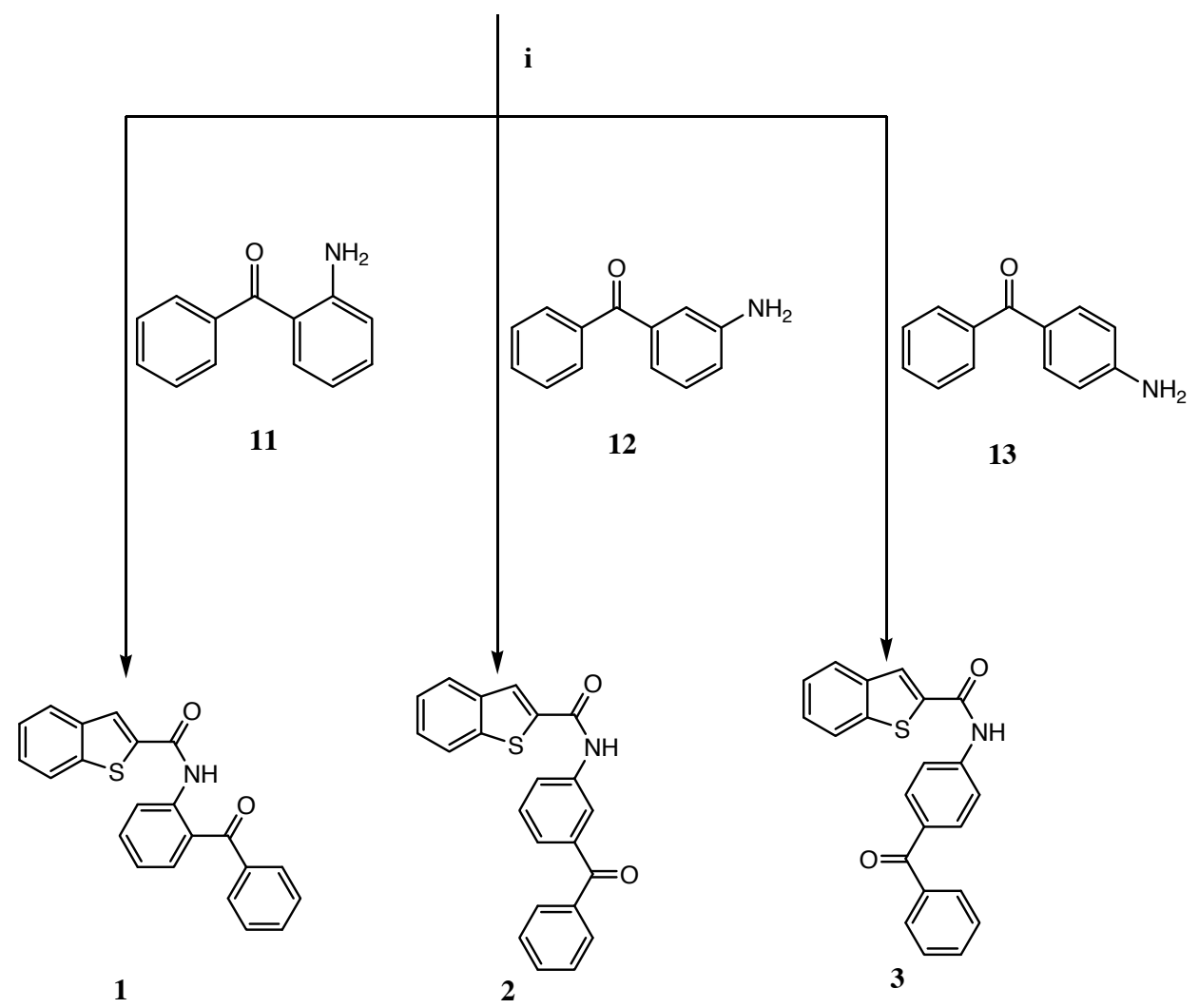

Scheme 1. Synthesis of benzothiophene carboxamide derivatives from unsubstituted aminobenzophenones. i) Fusion, $130-150{ }^{\circ} \mathrm{C}$.

noimidazole have been investigated for their potential hypolipidemic activity (Al-Qirim et al., 2009; Shahwan et al., 2010; Bosies et al., 1980; Sher and Ellsworth, 2004; Kopin et al., 2006; Dasseux and Oniciu, 2002). But to the best of our knowledge, a combination of benzothiophene with other groups of compounds such as benzophenone, aminopyridine, and aminoimidazole has not been investigated before as potential lipid-lowering agents.

Taking into consideration the correlation between hyperlipidemia and heart diseases, development of new agents with the potential capability to reduce elevated blood lipid levels is considered by medical authorities to be extremely important for the treatment and prevention of heart diseases. Therefore, this study aimed to evaluate the possible lipid-lowering activity of novel benzothiophene carboxamide derivatives of aminobenzophenone, aminopyridine, aminobenzimidazole, and aniline derivatives.

\section{Material and Methods}

\section{Chemical studies}

The benzothiophene carboxamide derivatives of aminobenzophenones, aminopyridines, and anilines, compounds 1-8, were synthesized in one step by melting benzothiophene-2-carbonyl chloride (10) with the corresponding aminobenzophe- 

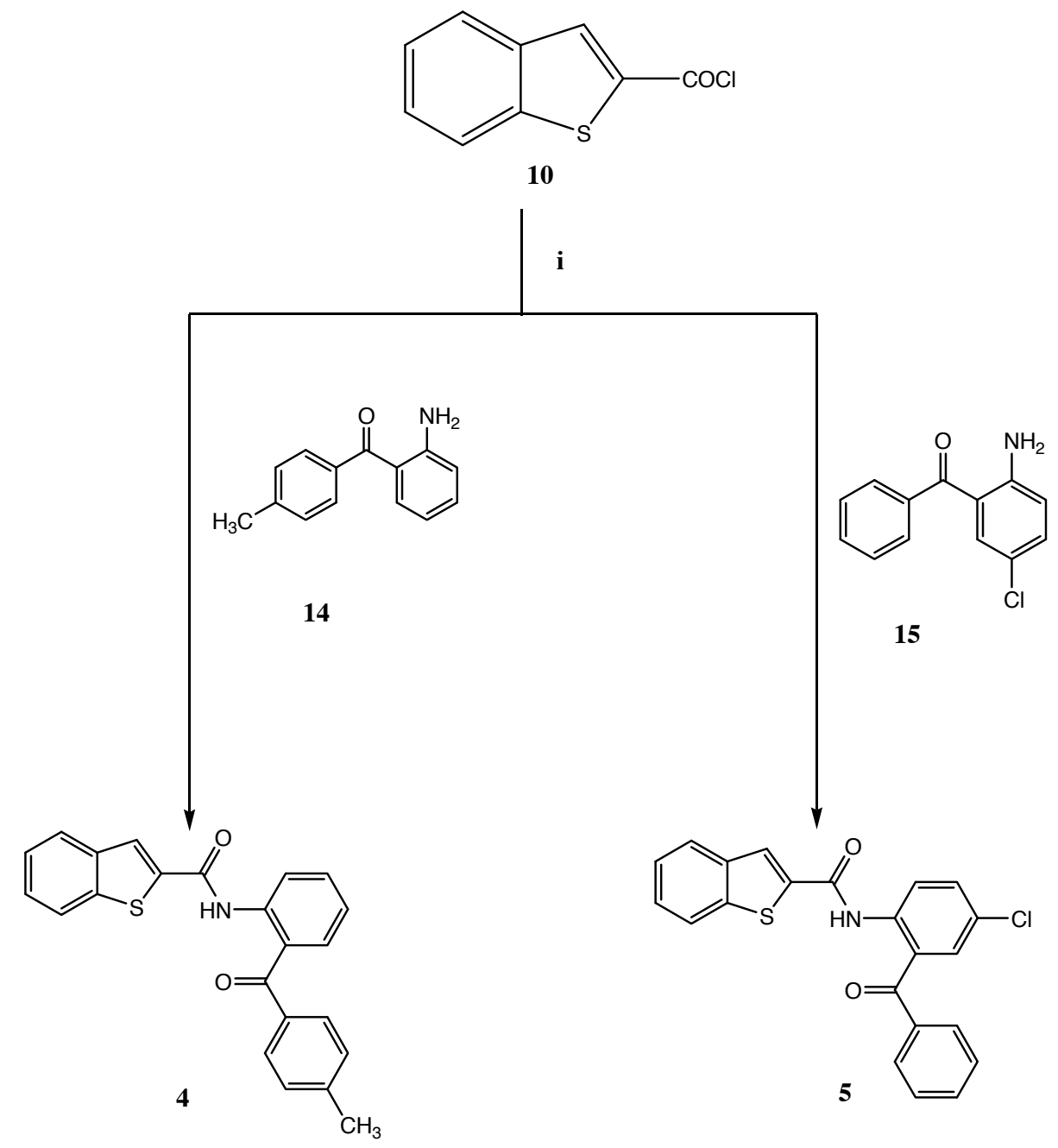

Scheme 2. Synthesis of benzothiophene carboxamide derivatives from substituted aminobenzophenones. i) Fusion, $130-150{ }^{\circ} \mathrm{C}$.

nones 11-15 at $130{ }^{\circ} \mathrm{C}$, as shown in Schemes 1 and 2, and with 2 - and 3-aminopyridines $\mathbf{1 6}$ and 17, and with $p$-chloroaniline $\mathbf{1 8}$ at $150{ }^{\circ} \mathrm{C}$ for $20 \mathrm{~min}$, according to Scheme 3. The mixtures of reactions were furthermore purified by using column chromatography to afford the desired compounds in high yields (75-90\%). Each compound was characterized by ${ }^{1} \mathrm{H}$ NMR, ${ }^{13} \mathrm{C}$ NMR, IR, elemental, and mass spectroscopic analyses, and was found to have the correct structure. Compound 9 was prepared by reaction of benzothiophene2-carbonyl chloride (10) with 2-aminobenzimidazole (19) in the presence of triethylamine in DMF at $160{ }^{\circ} \mathrm{C}$ (Scheme 3$)$.
Melting points were measured using a Gallenkamp melting point apparatus (Watford, UK) and are uncorrected. ${ }^{1} \mathrm{H}$ NMR and ${ }^{13} \mathrm{C}$ NMR spectra were collected on a Varian Oxford NMR ${ }^{300}$ spectrometer (Santa Clara, USA). The samples were dissolved in $\mathrm{CDCl}_{3}$ at a content of $0.3-0.7 \mathrm{wt}-\%$ and placed in 5-mm NMR tubes. High-resolution mass spectra (HRMS) were measured in the positive ion mode using the electrospray ion trap (ESI) technique by collision-induced dissociation on a Bruker Apex-4 (Tesla) instrument (Bremen, Germany). The samples were dissolved in acetonitrile, diluted in spray solution (methanol/ water, 5:4.9, v/v +0.1 formic acid) and infused 


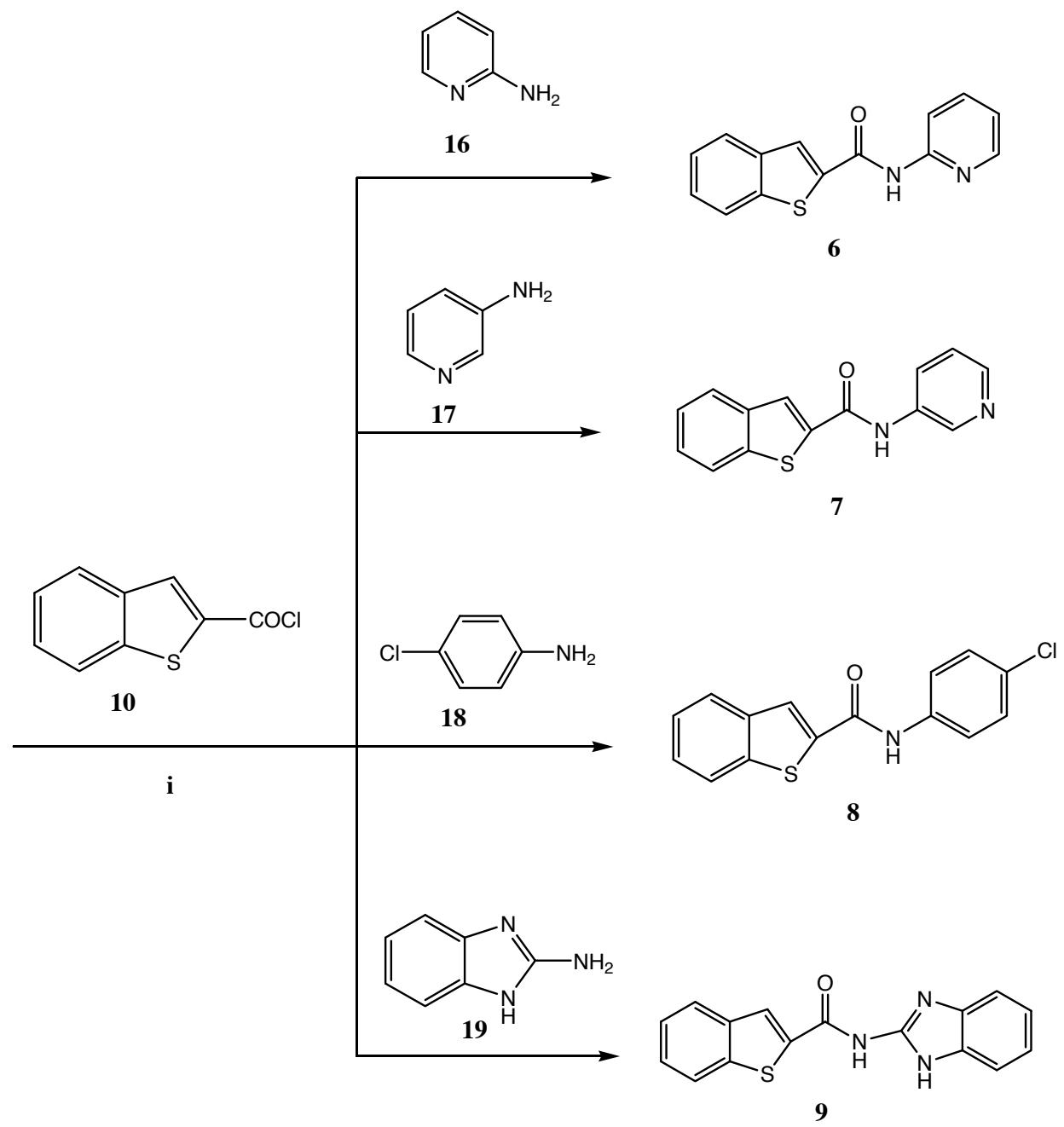

Scheme 3. Synthesis of benzothiophene carboxamide derivatives from aminopyridines, $p$-chloroaniline, and aminobenzimidazole. i) Fusion, $130-150{ }^{\circ} \mathrm{C}$ for compounds 16, 17, and 18, and DMF, triethylamine, reflux for compound 19.

using the syringe pump with a flow rate of $2 \mu \mathrm{l} /$ min. External calibration was conducted using an arginine cluster in the mass range $m / z=175-871$.

IR spectra were recorded using a Shimadzu IR affinity-1 spectrophotometer (Kyoto, Japan). The samples were dissolved in $\mathrm{CHCl}_{3}$ and analysed as thin solid films using $\mathrm{NaCl}$ plates. Analytical thin layer chromatography (TLC) was carried out using pre-coated aluminum plates and the bands were visualized by UV light (at 254 and/or $360 \mathrm{~nm}$ ). Elemental analysis was performed using an EuroVector elemental analyzer (Milan, Italy).
Chemicals and solvents were purchased from Sigma-Aldrich (St. Louis, MO, USA) and were used without further purification.

\section{Preparation of benzothiophene-2-carbonyl chloride (10)}

Benzothiophen-2-carboxylic acid $(1.0 \mathrm{~g}$, $5.6 \mathrm{mmol})$ was treated with thionyl chloride $(5 \mathrm{ml}$, $68.0 \mathrm{mmol}$ ). The reaction mixture was refluxed for $3 \mathrm{~h}$ at $130^{\circ} \mathrm{C}$, and then evaporated under reduced pressure. The product was washed twice with 
$10 \mathrm{ml}$ benzene to give the title compound as pale yellow solid (1.04 g, 94\%).

\section{Preparation of N-(2-benzoylphenyl)-} benzothiophene-2-carboxamide (1)

Benzothiophene-2-carbonyl chloride $(0.5 \mathrm{~g}$, $2.8 \mathrm{mmol})$ was treated with 2-aminobenzophenone $(1.11 \mathrm{~g}, 5.6 \mathrm{mmol})$. The mixture was heated at $150{ }^{\circ} \mathrm{C}$ on a hot plate until complete fusion and left for $20 \mathrm{~min}$. The resulting residue was then dissolved in $\mathrm{CHCl}_{3}$ and extracted from water, and the organic layer was purified by column chromatography using $\mathrm{CHCl}_{3}$ as eluent to afford the title compound as yellow solid $(1.44 \mathrm{~g}, 79 \%)$. - M.p. $174-176{ }^{\circ} \mathrm{C} .-\mathrm{R}_{\mathrm{f}}=0.66\left(100 \% \mathrm{CHCl}_{3}\right)$. $-{ }^{1} \mathrm{H}$ NMR $\left(\mathrm{CDCl}_{3}\right): \delta=12.10(1 \mathrm{H}$, br s, NHCO $)$, $8.80(1 \mathrm{H}, \mathrm{d}, J=8.4 \mathrm{~Hz}), 7.95(1 \mathrm{H}, \mathrm{s}, \mathrm{H}-3$ benzothiophene $), 7.80(1 \mathrm{H}, \mathrm{d}, J=8.6 \mathrm{~Hz}), 7.76(1$ $\mathrm{H}, \mathrm{d}, J=8.4 \mathrm{~Hz}), 7.65(1 \mathrm{H}, \mathrm{d}, J=8.2 \mathrm{~Hz}), 7.60$ $(1 \mathrm{H}, \mathrm{d}, J=8.2 \mathrm{~Hz}), 7.52(1 \mathrm{H}, \mathrm{d}, J=8.6 \mathrm{~Hz})$, $7.25(6 \mathrm{H}, \mathrm{m}), 7.1(1 \mathrm{H}, \mathrm{m}) .-{ }^{13} \mathrm{C}$ NMR $\left(\mathrm{CDCl}_{3}\right)$ : $\delta=198.1,160.4,150.3,139.8,135.8,134.3,133.4$, $130.3,130.1,128.5,128.1,127.7,126.4,124.3,122.3$, 121.6, 121.3, 120.7, 120.3, 119.7, 111.3, 103.2. - IR $(\mathrm{KBr}): v=3182,3051,1662,1627,1604,1585,1454$, 1323, 1300, 1242, 1165, 1153, 1072, 1049, 937, 918, $883,860,840 \mathrm{~cm}^{-1}$. - MS (ESI, positive mode): $m / z=358.08570[\mathrm{M}+\mathrm{H}]^{+}\left(\mathrm{C}_{22} \mathrm{H}_{16} \mathrm{NO}_{2} \mathrm{~S}\right.$ requires 358.08235). $-\mathrm{C}_{22} \mathrm{H}_{15} \mathrm{NO}_{2} \mathrm{~S}$ : calcd. C 73.93, H 4.23, N 3.92, S 8.97; found C 73.99, H 4.16, N 3.97, S 8.94.

\section{Preparation of $\mathrm{N}$-(3-benzoylphenyl)-}

benzothiophene-2-carboxamide (2)

Benzothiophene-2-carbonyl chloride $(0.5 \mathrm{~g}$, $2.8 \mathrm{mmol})$ was treated with 3 -aminobenzophenone $(0.5,2.5 \mathrm{mmol})$ for $25 \mathrm{~min}$ at $150{ }^{\circ} \mathrm{C}$, directly on a hot plate. The resulting residue was then purified by column chromatography using $\mathrm{CHCl}_{3}$ to give the desired compound as white solid $(0.15 \mathrm{~g}, 16 \%)$. - M.p. $156-159^{\circ} \mathrm{C} .-\mathrm{R}_{\mathrm{f}}=0.9$ $\left(\mathrm{CHCl}_{3} / \mathrm{CH}_{3} \mathrm{OH}, 80: 20\right) .-{ }^{1} \mathrm{H}$ NMR $\left(\mathrm{CDCl}_{3}\right): \delta=$ $10.75(1 \mathrm{H}$, br s, NHCO $), 8.40(1 \mathrm{H}, \mathrm{s}), 8.16(1 \mathrm{H}$, s, H-3 benzothiophene), $8.10(1 \mathrm{H}, \mathrm{d}, J=8.7 \mathrm{~Hz})$, $7.82-8.05(3 \mathrm{H}, \mathrm{m}), 7.65(1 \mathrm{H}, \mathrm{d}, J=8.2 \mathrm{~Hz}), 7.60$ $(1 \mathrm{H}, \mathrm{d}, J=8.2 \mathrm{~Hz}), 7.40-7.56(6 \mathrm{H}, \mathrm{m}) .-{ }^{13} \mathrm{C}$ NMR $\left(\mathrm{CDCl}_{3}\right): \delta=199.2,159.6,149.5,137.8,135.8$, 134.1, 133.6, 131.7, 130.4, 128.6, 128.1, 127.6, 126.2, 124.2, 121.9, 121.4, 121.1, 120.8, 120.3, 119.7, 111.6, 103.2. - IR (KBr): $v=3363,3062,1666,1643,1589$, $1546,1427,1315,975,871 \mathrm{~cm}^{-1}$. - MS (ESI, positive mode): $m / z=358.08570[\mathrm{M}+\mathrm{H}]^{+}\left(\mathrm{C}_{22} \mathrm{H}_{16} \mathrm{NO}_{2} \mathrm{~S}\right.$ requires 358.08235). $-\mathrm{C}_{22} \mathrm{H}_{15} \mathrm{NO}_{2} \mathrm{~S}$ : calcd. C 73.93, $\mathrm{H} 4.23$, N 3.92, S 8.97; found C 73.87, H 4.30, N 3.84, S 8.93 .

Preparation of $N$-(4-benzoylphenyl)benzothiophene-2-carboxamide (3)

Benzothiophene-2-carbonyl chloride $(0.5 \mathrm{~g}$, $2.8 \mathrm{mmol}$ ) was treated with 4-aminobenzophenone $(0.54 \mathrm{~g}, 2.7 \mathrm{mmol})$. The mixture was heated at $150{ }^{\circ} \mathrm{C}$, using a hot plate, and then the resulting residue was purified by recrystallization using $\mathrm{CHCl}_{3}$ and methanol to afford the title compound as yellow crystals $(0.81 \mathrm{~g}, 81 \%)$. - M.p. $198-202{ }^{\circ} \mathrm{C}$. $-\mathrm{R}_{\mathrm{f}}=0.52\left(\mathrm{CHCl}_{3} / \mathrm{CH}_{3} \mathrm{OH}, 90: 10\right) .-{ }^{1} \mathrm{H}$ NMR $\left(\mathrm{CDCl}_{3}\right): \delta=10.80(1 \mathrm{H}$, br s, NHCO $), 8.40(1 \mathrm{H}$, s, H-3 benzothiophene), $8.0(1 \mathrm{H}, \mathrm{d}, J=8.6 \mathrm{~Hz})$, $7.85(1 \mathrm{H}, \mathrm{d}, J=8.5 \mathrm{~Hz}), 7.78(2 \mathrm{H}, \mathrm{d}, J=8.2 \mathrm{~Hz})$, $7.70(2 \mathrm{H}, \mathrm{d}, J=8.2 \mathrm{~Hz}), 7.63(1 \mathrm{H}, \mathrm{d}, J=8.4 \mathrm{~Hz})$, $7.54(1 \mathrm{H}, \mathrm{d}, J=8.4 \mathrm{~Hz}), 7.42-7.52(5 \mathrm{H}, \mathrm{m}) .-{ }^{13} \mathrm{C}$ $\operatorname{NMR}\left(\mathrm{CDCl}_{3}\right): \delta=200.1,160.2,140.4,138.1,136.3$, 133.7, 133.3, 131.4, 130.2, 128.8, 128.1, 127.4, 126.8, 123.9, 121.5, 121.4, 121.2, 121, 120, 119.7, 111, 103. - IR (KBr): $v=3406,3055,1924,1639,1593,1527$, $1500,1404,1315,1284,1242,1184,1149,1122,925$, 844, 794, $740 \mathrm{~cm}^{-1}$. - MS (ESI, positive mode): $m / z=380.08253[\mathrm{M}+\mathrm{Na}]^{+}\left(\mathrm{C}_{22} \mathrm{H}_{15} \mathrm{NNaO}_{2} \mathrm{~S}\right.$ requires 380.08201). $-\mathrm{C}_{22} \mathrm{H}_{15} \mathrm{NO}_{2} \mathrm{~S}$ : calcd. $\mathrm{C} 73.93$, $\mathrm{H} 4.23, \mathrm{~N} 3.92$, S 8.97; found C 73.99, H 4.30, N $3.85, \mathrm{~S} 9.01$.

Preparation of N-[2-(4-methylbenzoyl) phenyl]-1benzothiophene-2-carboxamide (4)

Benzothiophene-2-carbonyl chloride $(0.5 \mathrm{~g}$, $2.8 \mathrm{mmol}$ ) was treated with 2-amino-4'-methylbenzophenone $(0.54 \mathrm{~g}, 2.5 \mathrm{mmol})$. The mixture was heated at $150{ }^{\circ} \mathrm{C}$ for $25 \mathrm{~min}$, and then the resulting residue was purified by recrystallization using $\mathrm{CHCl}_{3}$ and methanol to afford the title compound as yellow solid $(0.15 \mathrm{~g}, 16 \%)$. - M.p. $168-170{ }^{\circ} \mathrm{C} .-\mathrm{R}_{\mathrm{f}}=0.65\left(100 \% \mathrm{CHCl}_{3}\right)$. $-{ }^{1} \mathrm{H}$ NMR $\left(\mathrm{CDCl}_{3}\right): \delta=12.1(1 \mathrm{H}$, br s, NHCO $)$, $8.85(1 \mathrm{H}, \mathrm{d}, J=7.2 \mathrm{~Hz}), 8.05(1 \mathrm{H}, \mathrm{s}), 7.85(2 \mathrm{H}$, m), $7.65(3 \mathrm{H}, \mathrm{m}), 7.45(2 \mathrm{H}, \mathrm{m}), 7.33(2 \mathrm{H}, \mathrm{m})$, $7.15(2 \mathrm{H}, \mathrm{m}), 2.45(3 \mathrm{H}, \mathrm{s}) .-{ }^{13} \mathrm{C}$ NMR $\left(\mathrm{CDCl}_{3}\right)$ : $\delta=200.23,160.95,143.46,142.57,141.35,140.68$, $139.75,139.53,139.28,136.01,134.56,134.04$, $130.14,129.09,126.55,125.63,125.44,124.94$, 123.09, 122.76, 122.33, 121.30, 21.69. - IR (KBr): $v=3278,1674,1624,1600,1581,1523,1446$, 1319, 1292, 1261, 1246, 1165, 929, 833, $763 \mathrm{~cm}^{-1}$. - MS (ESI, positive mode): $\mathrm{m} / z=394.10135$ 
$[\mathrm{M}+\mathrm{Na}]^{+} \quad\left(\mathrm{C}_{23} \mathrm{H}_{17} \mathrm{NNaO}_{2} \mathrm{~S}\right.$ requires 394.09800). $-\mathrm{C}_{23} \mathrm{H}_{17} \mathrm{NO}_{2} \mathrm{~S}$ : calcd. C 74.37, H 4.61, N 3.77, S 8.63; found C 74.69, H 4.57, N 3.71, S 8.68.

Preparation of N-[2-(benzoyl-4-chlorophenyl)]benzothiophene-2-carboxamide (5)

Benzothiophene-2-carbonyl chloride $(0.5 \mathrm{~g}$, $2.8 \mathrm{mmol}$ ) was treated with 2 -amino-5-chlorobenzophenone $(0.57 \mathrm{~g}, 2.4 \mathrm{mmol})$. The mixture was heated at $150{ }^{\circ} \mathrm{C}$ for 20 min directly on a hot plate. The resulting residue was dissolved in $\mathrm{CHCl}_{3}$ and extracted from acidic water, then evaporated, and recrystallized from ethanol to afford the title compound as yellow crystals $(0.76 \mathrm{~g}, 77 \%)$. - M.p. $179-181{ }^{\circ} \mathrm{C} .-\mathrm{R}_{\mathrm{f}}=0.73\left(100 \% \mathrm{CHCl}_{3}\right)$. $-{ }^{1} \mathrm{H}$ NMR $\left(\mathrm{CDCl}_{3}\right): \delta=12.01(1 \mathrm{H}$, br s, NHCO $)$, $8.87(1 \mathrm{H}, \mathrm{d}, J=7.2 \mathrm{~Hz}), 8.05(1 \mathrm{H}, \mathrm{s}), 7.92(2$ $\mathrm{H}, \mathrm{m}), 7.75(2 \mathrm{H}, \mathrm{m}), 7.45-7.68(7 \mathrm{H}, \mathrm{m}) .-{ }^{13} \mathrm{C}$ NMR $\left(\mathrm{CDCl}_{3}\right): \delta=199.35,160.92,141.62,140.53$, $139.37,139.24,139.19,138.04,134.54,134.01$, $133.31,132.98,129.82,128.65,127.55,126.74$, 125.91, 125.49, 125.05, 123.95, 122.81, 122.78. - IR $(\mathrm{KBr}): v=1674,1631,1581,1523,1400,1319,1288$, 1242, 948, $756 \mathrm{~cm}^{-1}$. - MS (ESI, positive mode): $m / z=414.04338[\mathrm{M}+\mathrm{Na}]^{+}\left(\mathrm{C}_{22} \mathrm{H}_{14} \mathrm{ClNNaO}_{2} \mathrm{~S}\right.$ requires 414.04300). $-\mathrm{C}_{22} \mathrm{H}_{14} \mathrm{ClNO}_{2} \mathrm{~S}$ : calcd. C 67.43, H 3.60, N 3.57, S 8.18; found C 67.35, H 3.57, N 3.63, S 8.28.

Preparation of $\mathrm{N}$-(pyridin-2-yl)-benzothiophene2-carboxamide (6)

Benzothiophene-2-carbonyl chloride $(0.5 \mathrm{~g}$, $2.8 \mathrm{mmol})$ was treated with 2-aminopyridine $(0.24 \mathrm{~g}, 2.5 \mathrm{mmol})$. The mixture was heated at $150{ }^{\circ} \mathrm{C}$ for $15 \mathrm{~min}$ directly on a hot plate. The resulting residue was then purified by recrystallization using $\mathrm{CHCl}_{3}$ to afford the title compound as a white solid $(0.36 \mathrm{~g}, 58 \%)$. - M.p. $225-228{ }^{\circ} \mathrm{C}$. - $\mathrm{R}_{\mathrm{f}}=0.55\left(\mathrm{CHCl}_{3} / \mathrm{CH}_{3} \mathrm{OH}, 90: 10\right) .-{ }^{1} \mathrm{H}$ NMR $\left(\mathrm{CDCl}_{3}\right): \delta=12.55(1 \mathrm{H}$, br s, NHCO $), 8.95(1 \mathrm{H}$, $\mathrm{d}, J=7.2 \mathrm{~Hz}), 8.75(1 \mathrm{H}, \mathrm{s}), 8.22(2 \mathrm{H} \mathrm{m}), 7.95(1 \mathrm{H}$, $\mathrm{d}, J=7.2 \mathrm{~Hz}), 7.83(1 \mathrm{H}, \mathrm{d}, J=7.2 \mathrm{~Hz}), 7.32-7.42$ $(3 \mathrm{H}, \mathrm{m}) .-{ }^{13} \mathrm{C}$ NMR $\left(\mathrm{CDCl}_{3}\right): \delta=168.62,149.58$, 146.07,142.58, 140.02, 139.24, 137.37, 130.07,127.68, 126.73, 125.29, 122.60, 119.30, 117.49. - IR (KBr): $v=3070,3005,1681,1651,1608,1566,1500,1431$, 1330, 1292, 1238, 1211, 1153, 1041, 1006, $887 \mathrm{~cm}^{-1}$. - MS (ESI, positive mode): $\mathrm{m} / z=277.05138$ $[\mathrm{M}+\mathrm{Na}]^{+}\left(\mathrm{C}_{14} \mathrm{H}_{10} \mathrm{~N}_{2} \mathrm{NaOS}\right.$ requires 277.05179). $-\mathrm{C}_{14} \mathrm{H}_{10} \mathrm{~N}_{2} \mathrm{OS}$ : calcd. C 66.12, H 3.96, N 11.02, S 12.61; found C 66.20, H 3.90, N 11.11, S 12.68.
Preparation of $\mathrm{N}$-(pyridin-3-yl)-benzothiophene2-carboxamide (7)

Benzothiophene-2-carbonyl chloride $(0.25 \mathrm{~g}$, $1.4 \mathrm{mmol})$ was treated with 3-aminopyridine $(0.12 \mathrm{~g}, 1.3 \mathrm{mmol})$. The mixture was heated at $130^{\circ} \mathrm{C}$ for $22 \mathrm{~min}$ directly on a hot plate. The resulting residue was then purified by recrystallization using $\mathrm{CHCl}_{3}$ to afford the title compound as light gray solid $(0.32 \mathrm{~g}, 98 \%)$. - M.p. $265-269^{\circ} \mathrm{C}$. $-\mathrm{R}_{\mathrm{f}}=0.6\left(\mathrm{CHCl}_{3} / \mathrm{CH}_{3} \mathrm{OH}, 90: 10\right) .-{ }^{1} \mathrm{H} \mathrm{NMR}$ $\left(\mathrm{CDCl}_{3}\right): \delta=12.30$ ( $1 \mathrm{H}$, br s, NHCO), 9.45 (1 $\mathrm{H}, \mathrm{s}), 8.90(1 \mathrm{H}, \mathrm{d}, J=7.2 \mathrm{~Hz}), 8.70(1 \mathrm{H}, \mathrm{s}), 8.65$ $(1 \mathrm{H}, \mathrm{d}, J=7.2 \mathrm{~Hz}), 8.15(1 \mathrm{H}, \mathrm{d}, J=7.2 \mathrm{~Hz}), 8.05$ $(2 \mathrm{H}, \mathrm{m}), 7.50(2 \mathrm{H}, \mathrm{m}) .-{ }^{13} \mathrm{C}$ NMR $\left(\mathrm{CDCl}_{3}\right)$ : $\delta=166.51,146.09,144.18,143.40,142.70,139.63$, $139.03,136.41,133.21,132.28,132.16,130.98$, 130.50, 128.16. - IR (KBr): $v=3363,3062,1666$, $1643,1589,1546,1427,1315,975,871 \mathrm{~cm}^{-1}$. - MS (ESI, positive mode): $m / z=255.05138[\mathrm{M}+\mathrm{H}]^{+}$ $\left(\mathrm{C}_{14} \mathrm{H}_{11} \mathrm{~N}_{2} \mathrm{OS}\right.$ requires 255.05159). $-\mathrm{C}_{14} \mathrm{H}_{10} \mathrm{~N}_{2} \mathrm{OS}$ : calcd. C 66.12, H 3.96, N 11.02, S 12.61; found C 66.04, H 4.02, N 10.93, S 12.66.

\section{Preparation of $\mathrm{N}$-(4-chlorophenyl)- benzothiophene-2-carboxamide (8)}

Benzothiophene-2-carbonyl chloride $(0.5 \mathrm{~g}$, $2.8 \mathrm{mmol})$ was added to $p$-chloroaniline $(0.32 \mathrm{~g}$, $2.5 \mathrm{mmol})$. The mixture was heated on a hot plate at $130-140{ }^{\circ} \mathrm{C}$ for $20 \mathrm{~min}$. The resulting residue was purified by recrystallization using $\mathrm{CHCl}_{3}$ to afford the title compound as a white solid $(0.37 \mathrm{~g}$, $49 \%)$ - M.p. $215-220^{\circ} \mathrm{C}$. $-\mathrm{R}_{\mathrm{f}}=0.51(100 \%$ $\left.\mathrm{CHCl}_{3}\right) .-{ }^{1} \mathrm{H}$ NMR $\left(\mathrm{CDCl}_{3}\right): \delta=10.70(1 \mathrm{H}$, br s, NHCO $), 8.37$ (1 H, s), $8.01-8.08(2 \mathrm{H}, \mathrm{m}), 7.83$ $(2 \mathrm{H}, \mathrm{d}, J=9 \mathrm{~Hz}), 7.43-7.53(4 \mathrm{H}, \mathrm{m}) .-{ }^{13} \mathrm{C}$ NMR $\left(\mathrm{CDCl}_{3}\right): \delta=165.62,145.78,144.89,144.28$, $142.28,139.36,139.15,138.85,133.89,132.83$, 131.81, 130.67, 130.31, 128.08, 127.05. - IR (KBr): $v=3360,3093,1647,1593,1527,1492,1431,1400$, 1307, 1242, 1184, 1095, 1014, 821, 790, $725 \mathrm{~cm}^{-1}$. - MS (ESI, positive mode): $\mathrm{m} / z=288.01716$ $[\mathrm{M}+\mathrm{H}]^{+} \quad\left(\mathrm{C}_{15} \mathrm{H}_{11} \mathrm{ClNOS}\right.$ requires 288.01664). $-\mathrm{C}_{15} \mathrm{H}_{10}$ ClNOS: calcd. C 62.61, H 3.50, N 4.87, S 11.14; found C 62.54, H 3.43, N 4.95, S 11.22.

\section{Preparation of $\mathrm{N}-(1 \mathrm{H}$-benzimidazol-2-yl)- benzothiophene-2-carboxamide (9)}

Benzothiophene-2-carbonyl chloride (1.33 g, $7.5 \mathrm{mmol}$ ) was treated with 2-aminobenzimidazole $(0.9 \mathrm{~g}, 6.8 \mathrm{mmol})$ in $15 \mathrm{ml} \mathrm{DMF}$ in the presence of 
triethylamine $(2.0 \mathrm{ml}, 7.5 \mathrm{mmol})$. The mixture was refluxed for $48 \mathrm{~h}$. DMF was removed by evaporation under reduced pressure, and the resulting residue was redissolved in $\mathrm{CHCl}_{3}$ and extracted with acidic water. The organic layer, after drying over anhydrous $\mathrm{Na}_{2} \mathrm{SO}_{4}$, was purified by column chromatography using $\mathrm{CHCl}_{3}$ as eluent to afford the title compound as a white solid $(0.3 \mathrm{~g}, 27 \%)$. - M.p. $262-268{ }^{\circ} \mathrm{C} .-\mathrm{R}_{\mathrm{f}}=0.82\left(\mathrm{CHCl}_{3} / \mathrm{CH}_{3} \mathrm{OH}\right.$, 85:15). $-{ }^{1} \mathrm{H}$ NMR $\left(\mathrm{CDCl}_{3}\right): \delta=12.00(1 \mathrm{H}$, br s, NHCO $), 10.23(1 \mathrm{H}$, br s), $8.65(1 \mathrm{H}, \mathrm{s}), 8.00(2 \mathrm{H}$, $\mathrm{t}, J=7.5 \mathrm{~Hz},), 7.60(2 \mathrm{H}, \mathrm{m}),, 7.45(2 \mathrm{H}, \mathrm{m}), 7.30$ $(2 \mathrm{H}, \mathrm{m}) .-{ }^{13} \mathrm{C}$ NMR $\left(\mathrm{CDCl}_{3}\right): \delta=163.51,146.23$, $141.82,139.38,139.15,139.01,138.96,138.75$, $129.73,129.53,127.75,126.42,125.78,124.58$, 123.47, 113.57. - IR (KBr): $v=3344,3093,1639$, 1597, 1558, 1516, 1462, 1431, 1346, 1276, 1249, 1219, 1180, 1157, 1099, $883 \mathrm{~cm}^{-1}$. - MS (ESI, positive mode): $m / z=316.06228[\mathrm{M}+\mathrm{Na}]^{+}\left(\mathrm{C}_{16} \mathrm{H}_{11} \mathrm{~N}_{3} \mathrm{NaOS}\right.$ requires 316.06283). $-\mathrm{C}_{16} \mathrm{H}_{11} \mathrm{~N}_{3} \mathrm{OS}$ : calcd. C 65.51, H 3.78, N 14.32, S 10.93; found C 65.42, H 3.86, N 14.39, S 10.86 .

\section{Pharmacological studies}

Triton WR-1339 was obtained from Sigma-Aldrich. The rest of the chemicals (fine super grade) were purchased from Acros Organics (Amman, Jordan).

\section{Animals and treatments}

Sixty four adult male Wistar rats, weighing around $180 \mathrm{~g}$, bred in the animal care centre of Faculty of Pharmacy, Al-Zaytoonah Private University, Amman, Jordan, were provided ad libitum access only to tap water throughout the experimental duration $(24 \mathrm{~h})$. Rats were maintained in a 12-h light-dark cycle under constant humidity and $(22 \pm 2){ }^{\circ} \mathrm{C}$. All experiments were performed in accordance with the Guidelines of Animal Welfare Committee of the University.

\section{Triton WR-1339 model of hyperlipidemia}

Triton WR-1339 was dissolved in DMSO and administered intraperitoneally $(0.6 \mathrm{ml})$ to the rats $(300 \mathrm{mg} / \mathrm{kg}$ body weight) in order to induce hyperlipidemia.

\section{Experimental design}

Overnight fasted rats were randomly divided into eight groups of eight animals each. The first group, serving as normal control group (NCG), received an intraperitoneal administration of normal saline; the second hyperlipidemic plus $4 \%$ DMSO control group (TDCG) received an intraperitoneal injection of Triton WR-1339 dissolved in $4 \%$ DMSO. In the third group (C3) animals were intraperitoneally injected with Triton WR1339 , followed by an intragastric administration of compound 3 (15 mg/kg body weight) dissolved in $4 \%$ DMSO. The rats of the fourth group (C6) were also intraperitoneally injected with Triton WR-1339, followed by an intragastric administration of compound $6(15 \mathrm{mg} / \mathrm{kg}$ body weight) dissolved in $4 \%$ DMSO. At the same time the rats of the fifth group (C7) were also intraperitoneally injected with Triton WR-1339, followed by an intragastric administration of compound $7(15 \mathrm{mg} /$ $\mathrm{kg}$ body weight) dissolved in $4 \%$ DMSO. In the sixth group (C8) animals were intraperitoneally injected with Triton WR-1339, followed by an intragastric administration of compound $8(15 \mathrm{mg} /$ $\mathrm{kg}$ body weight) dissolved in $4 \%$ DMSO. The rats of the seventh group (C9) were also intraperitoneally injected with Triton WR-1339, followed by an intragastric administration of compound 9 ( $15 \mathrm{mg} / \mathrm{kg}$ body weight) dissolved in 4\% DMSO. The last group (TDFG) was also intraperitoneally injected with Triton WR-1339 and intragastrically treated with bezafibrate $(100 \mathrm{mg} / \mathrm{kg}$ body weight) dissolved in $4 \%$ DMSO.

After $12 \mathrm{~h}$ of treatments, animals were anaesthetized with diethyl ether, and blood was collected from the renal artery. The blood samples were immediately centrifuged $(1500 \times \mathrm{x}$ for $10 \mathrm{~min})$, and the plasma was used for lipid analysis by an enzymatic method with an automatic analyzer (Model Erba XL-300, Mannheim, Germany).

\section{Statistical analysis}

Results were expressed as means \pm SD. Data obtained were analysed using the Student's t-test, and differences with $p<0.05$ were considered statistically significant.

\section{Results}

\section{Induction of hyperlipidemia by Triton WR-1339}

The plasma total cholesterol (TC), triglyceride (TG), high-density lipoprotein-cholesterol (HDLC), and low-density lipoprotein-cholesterol (LDLC) levels of all groups treated for $12 \mathrm{~h}$ are shown 
in Fig. 1. Triton WR-1339 caused a significant increase in plasma TC, TG and LDL-C $(p<0.0001)$ levels in the hyperlipidemic $+4 \%$ DMSO control group (TDCG), $12 \mathrm{~h}$ after Triton WR-1339 administration in comparison with the normal control group (NCG).

In fact, the increase of plasma TC concentration in TDCG was $45 \%$ after $12 \mathrm{~h}$ as compared to NCG. The TG level in TDCG was also elevated by $824 \%$ after $12 \mathrm{~h}$. At the same time the LDL-C level in TDCG was also elevated by $185 \%$ after $12 \mathrm{~h}$ as compared to NCG, while a significant $(p<$ $0.0001)$ decrease in the HDL-C level occurred $12 \mathrm{~h}$ after Triton WR-1339 injection.

\section{Effect of compounds $\mathbf{3}, \mathbf{6}, \mathbf{7}, \mathbf{8}, \mathbf{9}$, and bezafibrate} on rat plasma lipid profile

The plasma TC, TG, HDL-C, and LDL-C levels of TDFG-, compounds 3-, 7-, 8-, and 9-treated rats after $12 \mathrm{~h}$ are shown in Table I. Importantly, the elevated plasma TG levels produced by Triton WR-1339 administration were significantly $(p<$ 0.0001 ) suppressed in TDFG- by $77 \%$, in compound 6- by $73 \%$, in compound 7 - by $49 \%$, and in compound 9 -treated rats by $81 \%$ after $12 \mathrm{~h}$, with respect to the hyperlipidemic control TDCG.

The HDL-C levels were significantly increased after $12 \mathrm{~h}$ by $44 \%, 53 \%$, and $37 \%(p<0.0001)$ in compounds 6-, 7-, and 9-treated rats, respectively, and $16 \%(p<0.01)$ in TDFG-treated rats compared to the hyperlipidemic control TDCG (Table I).

With the exception of compound 9-treated rats, none of the treated groups showed a significant

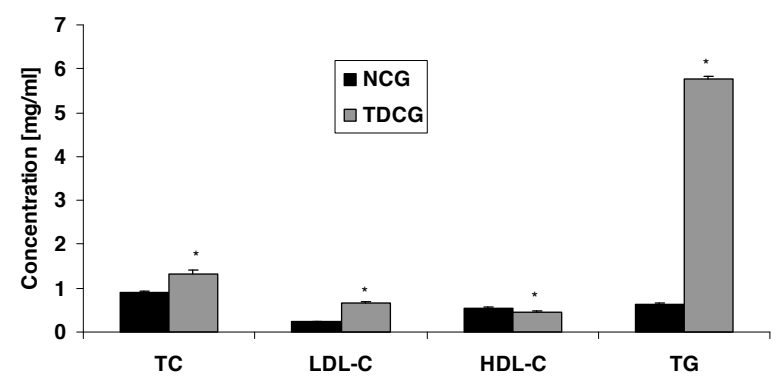

Fig. 1. Effect of Triton WR-1339 on plasma lipid levels after $12 \mathrm{~h}$. Values are means \pm SD from eight animals in each group. NCG, control group; TDCG, hyperlipidemic control group; TC, total cholesterol; TG, triglyceride; HDL-C, high-density lipoprotein-cholesterol; LDL-C, low-density lipoprotein-cholesterol. " $p<0.0001$.

reduction in plasma TC levels after $12 \mathrm{~h}$ (Table I). In fact, it was found that the TC level was reduced by $8 \%$ after $12 \mathrm{~h}$ in compound 9- compared to TDCG-treated rats.

After $12 \mathrm{~h}$ of treatment, LDL-C levels were lowered by $44 \%(p<0.0001)$ in compound 9-, and $13 \%, 10 \%$, and $15 \%(p<0.01)$ in compounds 6 -, 7 -, and TDFG-treated rats, respectively, compared to the hyperlipidemic control group TDCG (Table I).

No significant differences in TC, TG, HDL-C, and LDL-C levels were observed in compounds 3- and 8-treated rats compared to TDCG-treated rats.

\section{Discussion}

The results of the current study showed the potential hypolipidemic effect of compounds $\mathbf{6}$,

Table I. Effect of the novel compounds 3, 6, 7, 8,9 and bezafibrate on plasma lipid levels in Triton WR-1339-induced hyperlipimic rats after $12 \mathrm{~h}$.

\begin{tabular}{lcccc}
\hline Lipid profile & TC $[\mathrm{mg} / \mathrm{ml}]$ & TG $[\mathrm{mg} / \mathrm{ml}]$ & HDL-C $[\mathrm{mg} / \mathrm{ml}]$ & LDL-C [mg/ml] \\
\hline TDCG & $1.32 \pm 0.08$ & $5.76 \pm 0.07$ & $0.45 \pm 0.03$ & $0.66 \pm 0.03$ \\
C3 & $1.29 \pm 0.04$ & $5.80 \pm 0.09$ & $0.46 \pm 0.02$ & $0.65 \pm 0.02$ \\
C6 & $1.21 \pm 0.06$ & $1.56 \pm 0.07^{\mathrm{b}}$ & $0.65 \pm 0.03^{\mathrm{b}}$ & $0.58 \pm 0.04^{\mathrm{a}}$ \\
C7 & $1.35 \pm 0.004$ & $2.91 \pm 0.05^{\mathrm{b}}$ & $0.69 \pm 0.03^{\mathrm{b}}$ & $0.60 \pm 0.04^{\mathrm{a}}$ \\
C8 & $1.31 \pm 0.05$ & $5.73 \pm 0.07$ & $0.44 \pm 0.02$ & $0.67 \pm 0.02$ \\
C9 & $1.21 \pm 0.03^{\mathrm{a}}$ & $1.09 \pm 0.03^{\mathrm{b}}$ & $0.62 \pm 0.03^{\mathrm{b}}$ & $0.37 \pm 0.03^{\mathrm{b}}$ \\
TDFG & $1.35 \pm 0.03^{\mathrm{a}}$ & $1.33 \pm 0.04^{\mathrm{b}}$ & $0.52 \pm 0.03^{\mathrm{a}}$ & $0.56 \pm 0.01^{\mathrm{a}}$ \\
\hline
\end{tabular}

Values are means \pm SD from eight animals in each group.

TDCG, hyperlipidemic + 4\% DMSO control group; C3, compound $3+4 \%$ DMSO; C6, compound $6+4 \%$ DMSO; C7, compound $7+4 \%$ DMSO; C8, compound 8 + 4\% DMSO; C9, compound $9+4 \%$ DMSO; TDFG, bezafibrate + 4\% DMSO; TC, total cholesterol; TG, triglyceride; HDL-C, high-density lipoprotein-cholesterol; LDL-C, lowdensity lipoprotein-cholesterol.

Compounds 3, 6, 7, 8, 9 and TDFG are compared with TDCG.

${ }^{\mathrm{a}} p<0.01 ;{ }^{\mathrm{b}} p<0.0001$. 
7, and 9 (Table I) in Triton WR-1339-induced hyperlipidemic rats. Compounds 6, 7, and 9 significantly reduced serum TG and increased serum HDL levels.

Triton WR-1339-induced hyperlipidemic rats have been widely used as model to investigate the potential hypolipidemic effect of synthesized and naturally derived compounds (Paoletti, 1962; Kalopissis et al., 1980; Khanna et al., 1992; Frishman, 1998). It has been reported that hyperlipidemia could be induced by parenteral administration of Triton WR-1339 to adult rats. The peak plasma TC and TG levels were reached at $20 \mathrm{~h}$ followed by a decline to normal values (Schurr et al., 1972). In our study, the same model showed a similar pattern of lipid profile changes $12 \mathrm{~h}$ after Triton WR-1339 administration (Fig. 1).

Clearly, the results in our hands showed that compounds 6, 7, and 9 at a dose of $15 \mathrm{mg} / \mathrm{kg}$ body weight were able to significantly decrease serum TG levels.

Yamamoto and his colleagues (1984) reported that the large decrease in plasma HDL-C levels due to Triton WR-1339 injection results mostly from a progressive displacement of the apo A-1 protein from the HDL surface without loss of lipid. Meanwhile the large increase in plasma TG levels due to Triton administration results mostly from an increase of very low-density lipoprotein (VLDL) secretion by the liver accompanied by strong reduction of VLDL and LDL catabolism (P'erez et al., 1999).

Accordingly, given that the portion of TG in VLDL is persistently higher than that of cholesterol, it is not unexpected that the hypolipidemic activity of compounds 6,7 , and 9 was significantly higher for TG than for cholesterol. This result suggests that the catabolism of B-lipoproteins could be, at least partially, restored by our compounds as hypothesized by many works with other lipidlowering agents (Khanna et al., 2002; Malloy and Kan, 1994).

In addition, compounds 6, 7, and 9 increased the HDL level, which is known for its preventive role against atherogenesis. HDL also promotes substantial cholesterol egress from the liver by facilitating the mobilization of TG and cholesterol from plasma to liver where it undergoes catabolism and then is eliminated in the form of bile acids (Anila and Vijayalakshmi, 2002; Staels et al., 1998).
Promisingly, administration of compounds 6, 7, and 9 at a dose of $15 \mathrm{mg} / \mathrm{kg}$ body weight after $12 \mathrm{~h}$ of Triton injection is more significant than the reduction induced by bezafibrate administration at a dose of $100 \mathrm{mg} / \mathrm{kg}$ body weight, which in this study has been used as standard reference hypolipidemic drug. Furthermore, TC levels were not significantly changed which agrees with the mechanism of action of fibrates in that their TClowering activity is not strongly marked, but the TG decreasing effect of them is very impressive

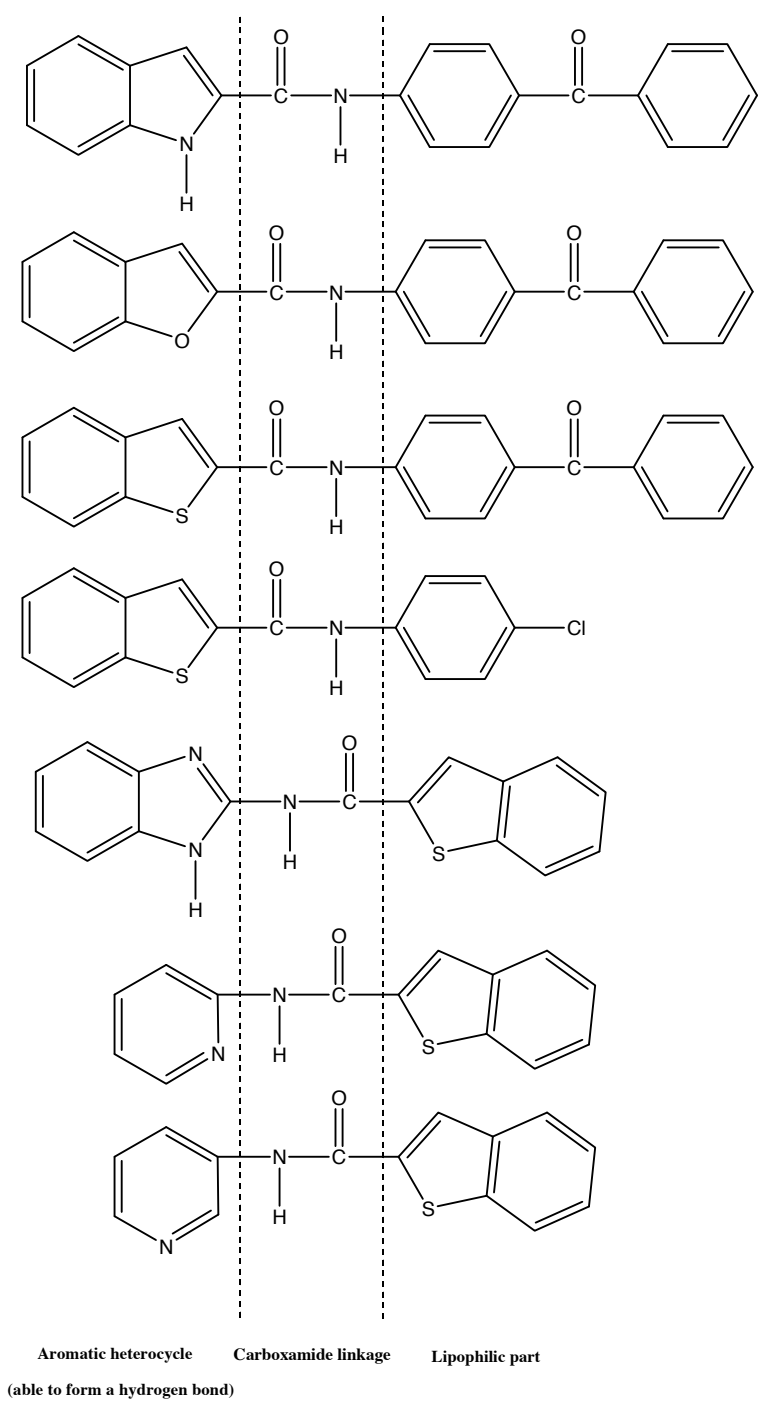

Fig. 2. Active and inactive carboxamide derivatives and the three essential components determining their activity. 
especially by stimulation of the gene expression of lipoprotein lipase (Staels et al., 1998).

The findings of the current study are compatible with our previously published data (Al-Qirim et al., 2009; Shahwan et al., 2010), which highlighted that introducing a hydrogen bond between aromatic heterocyclic rings and the target(s) is essential for the biological activity (Fig. 2). It was noticed that replacing indole and benzofuran by benzothiophene will abolish the activity even though the lipophilic moiety is conserved. On the other hand, the activity was retained when imidazole and pyridine are introduced into the compounds instead of indole and benzofuran. From these observations, we concluded that the presence of an aromatic heterocycle ring which is able to form a hydrogen bond is crucial for the biological activity (Fig. 2).

In addition, it seems that the presence of a large lipophilic moiety is also important for the

Al-Qirim T., Shahwan M., Shattat G., Al-Hiari Y., Abu Sheikha G., and Zaidi S. (2009), Pharmacological evaluation of novel indole-2-carboxamides as potent lipid-lowering agents in Triton-WR1339-induced hyperlipidemic rats. Z. Naturforsch. 64c, 619-625.

Anila L. and Vijayalakshmi N. R. (2002), Flavonoids from Emblica officinalis and Mangifera indica - effectiveness for dyslipidemia J. Ethnopharmacol. 79, $81-87$.

Bosies E., Heerdt R., Kuknle H. F., Schmidt F. H., and Stach H. (1980), Hypoglycemically and hypolipidemically active derivatives of phenylalkane carboxylic acids. US Patent 150207.

Charles D. J., Mary G. J., Andrew J. P., Mary K. P., Larry J. B., Allen R. T., Julie F. F., and James A. C. (1984), Antiestrogens. 2. Structure-activity studies in a series of 3-aroyl-2-arylbenzo[b]thiophene derivatives leading to [6-hydroxy-2-(4-hydroxyphenyl)benzo[ $b]$ thien3-yl]-[4-[2-(1-piperidinyl)ethoxy]phenyl] methanone hydrochloride (LY 156758), a remarkably effective estrogen antagonist with only minimal intrinsic estrogenicity. J. Med. Chem. 27, 1057-1066.

Dasseux J.-L. H. and Oniciu C. D. (2002), Aliphatic, aromatic, and heterocyclic ketone compounds and compositions for cholesterol management and related uses. US Patent 325529.

Frick M. H., Elo O., Haapa K., Heinonen O. P., Heinsalmi P., Helo P., Huttunen J. K., Kaitaniemi P., Koskinen P., Manninen V., Mäenpää H., Mälkönen M., Mänttäri M., Norola S., Pasternack A., Pikkarainen J., Romo M., Sjöblom T., and Nikkilä E. A. (1987), Helsinki heart study: primary-prevention trial with gemfibrozil in middle aged men with dyslipidemia. Safety of treatment, changes in risk factors, and in- biological activity. So when benzothiophene was used to replace benzophenone the activity was maintained whereas the activity was dramatically decreased when it was replaced by small lipophilic rings. This could be explained by ring equivalency between benzothiophene and benzophenone (Fig. 2).

Overall, these preliminary observations lead us to conclude that the presence of a large lipophilic moiety, carboxamide linker along with a heterocylic ring (able to form hydrogen bonds) are three important requirements to obtain hypolipidemic activity.

In conclusion, compounds $\mathbf{6}, \mathbf{7}$, and 9 were shown to improve the lipid profile in Triton-induced hyperlipidemic rats. The results are highly promising but more studies are necessary to elucidate the exact mechanism of action of these novel compounds as lipid-lowering agents and to clarify their structure-activity relationship.

cidence of coronary heart disease. N. Engl. J. Med. 317, $1237-1245$.

Frishman W. H. (1998), Biologic markers as predictors of cardiovascular disease. Am. J. Med. 104, 18S-27S.

Goldstein J. L., Hazzard W. R., Schrott H. G., Bierman E. L., and Motulsky A. G. (1973), Hyperlipidemia in coronary heart disease I. Lipid levels in 500 survivors of myocardial infarction. J. Clin. Invest. 52, 1533-1543.

Kalopissis A.-D., Griglio S., Malewiak M.-I., and Rosen R. (1980), Effect of a high-fat diet on rat very low density lipoprotein secretion. Biochim. Biophys. Acta 620, 111-119.

Khanna A. K., Chauder R., Chandan S., Srivastava A. K., and Kapoor N. K. (1992), Hypolipidemic activity of Achyranthus aspera Linn. in normal and Triton induced hyperlipimic rats. Indian J. Exp. Biol 30, $128-130$.

Khanna A. K., Rizvi F., and Chander R. (2002), Lipid lowering activity of Phyllanthus niruri in hyperlipimic rats. J. Ethnopharmacol. 82, 19-22.

Kopin A. S., Carey M., and Wang D. (2006), Altering intestinal motility and absorption of hydrophobic compounds through the use of agonists and/or antagonists of the cholecystokinin- 1 receptor. US Patent 224869.

Malloy M. J. and Kan J. P. (1994), Medical management of hyperlipidemic states. Adv. Int. Med. 39, 603-631.

Paoletti R. (1962), Comparative studies on hypocholesterolemic agents. Am. J. Clin. Nutr. 10, 277-284.

P'erez C., Canal J. R., Campello J. E., Adelaida R., and Torres M. D. (1999), Hypotriglyceridemic activity of Ficus carica leaves in experimental hypertriglyceridemic rats. Phytother. Res. 13, 188-191. 
Raasch R. H. (1988), Hyperlipidemias in applied therapeutics. In: The Clinical Use of Drugs (Young L. Y. and Koda-Kimble M. A., eds.). Edwards Brothers, Ann Arbor, MI, USA, pp. 1743-1745.

Rubins H. B., Robins S. J., Collins D., Fye C. L., Anderson J. W., Elam M. B., Faas F. H., Linares E., Schaefer E. J., Schectman G., Wilt T. J., and Wittes J. (1999), Gemfibrozil for the secondary prevention of coronary heart disease in men with low levels of highdensity lipoprotein cholesterol. N. Engl. J. Med. 341, 410-418.

Schoonjans K., Staels B., and Auwerx J. (1996), Role of the peroxisome proliferator-activated receptor (PPAR) in mediating the effects of fibrates and fatty acids on gene expression. J. Lipid Res. 37, 907-925.

Schotz M. C., Scanu A., and Page I. H. (1957), Effect of Triton on lipoprotein lipase of rat plasma. Am. J. Physiol. 188, 399-402.

Schurr P. E., Schultz J. R., and Parkinson T. M. (1972), Triton induced hyperlipidaemia in rats as an animal model for screening hypolipidaemic drugs. Lipids 7 , 69-74.

Shahwan M., Shattat G., Al-Qirim T., Abu Sheikha G., El-Huneidi W., Jarab A., and AL-Najdawi M. (2010), Synthesis and pharmacological evaluation of novel substituted and unsubstituted $N$-(benzoylphenyl) $-1 H$ indole-2-carboxamides as potent antihypertriglyceridemic agents. Z. Naturforsch. 65c, 309-316.

Sher P. M. and Ellsworth B. A. (2004), Triglyceride and triglyceride-like prodrugs of glycogen phosphorylase inhibiting compounds. US Patent 140474.

Staels B., Dallongville J., Auwerx J., Schoonjans K., Leitersdorf E., and Fruchart J. C. (1998), Mechanism of action of fibrates on lipid and lipoprotein metabolism. Circulation 98, 2088-2093.

Yamamoto K., Byrne R., Edelstein C., Shen B., and Scanu A.M. (1984), In vitro effect of Triton WR-1339 on canine plasma high density lipoproteins. J. Lipid Res. 25, 770-779. 